\title{
An Incremental Radial Basis Function Network Based on Information Granules and Its Application
}

\author{
Myung-Won Lee and Keun-Chang Kwak \\ Department of Control and Instrumentation Engineering, Chosun University, 375 Seosuk-dong, Dong-gu, \\ Gwangju 501-759, Republic of Korea \\ Correspondence should be addressed to Keun-Chang Kwak; kwak@chosun.ac.kr
}

Received 29 June 2016; Accepted 22 August 2016

Academic Editor: Toshihisa Tanaka

Copyright (C) 2016 M.-W. Lee and K.-C. Kwak. This is an open access article distributed under the Creative Commons Attribution License, which permits unrestricted use, distribution, and reproduction in any medium, provided the original work is properly cited.

\begin{abstract}
This paper is concerned with the design of an Incremental Radial Basis Function Network (IRBFN) by combining Linear Regression (LR) and local RBFN for the prediction of heating load and cooling load in residential buildings. Here the proposed IRBFN is designed by building a collection of information granules through Context-based Fuzzy C-Means (CFCM) clustering algorithm that is guided by the distribution of error of the linear part of the LR model. After adopting a construct of a LR as global model, refine it through local RBFN that captures remaining and more localized nonlinearities of the system to be considered. The experiments are performed on the estimation of energy performance of 768 diverse residential buildings. The experimental results revealed that the proposed IRBFN showed good performance in comparison to LR, the standard RBFN, RBFN with information granules, and Linguistic Model (LM).
\end{abstract}

\section{Introduction}

During the past few decades, we have witnessed a rapid growth in the number and variety of applications of fuzzy logic, neural networks, and evolutionary computing as a framework of computational intelligence [1-4]. We especially shall concentrate on incremental construction of Radial Basis Function Network (RBFN) with the aid of information granules. In general, we design with the simplest linear models and then refine such linear models by incorporating additional nonlinear model in system modeling. The commonly used method becomes Linear Regression (LR) model [5]. If LR model appears to be insufficient to predict, further refinements are implemented. This concept is a strong factor motivating the development of the incremental models. The effectiveness and superiority of this model have been demonstrated in the previous work. The incremental model introduced by Pedrycz and Kwak [6] represented a nonlinear and complex characteristic more effectively than conventional models. There are several advantages of this approach. First, a commonly used framework of LR has been used. The nonlinear behavior of the system could be confined to some limited regions of the input space and by adding only a few patches in these regions becomes practically relevant and conceptually justifiable. Furthermore, it has established a comprehensive design platform offering a complete setby-step procedure of the construction of the incremental model [7]. The clustering technique used in the design of incremental model is based on Context-based Fuzzy CMeans (CFCM) clustering algorithm [8]. This clustering algorithm generates information granules in the form of fuzzy sets and estimate clusters by preserving the homogeneity of the clustered data points associated with the input and output variables. In contrast to the context-free clustering methods [9-12], context-based fuzzy clustering is performed with the use of the contexts produced in output space. The effectiveness of the CFCM clustering has been successfully demonstrated in the previous works [13-16].

In this paper, we design the variant model with the fundamental idea of incremental model. For this purpose, we design an Incremental Radial Basis Function Network (IRBFN) by incorporating LR and local RBFN for accurate quantitative prediction of energy performance of residential buildings. We adopt a design of a LR as global model and 
refine it through local RBFN that captures remaining and more localized nonlinearities of the system with the aid of information granulation. Here the learning methods of local RBFN are performed by LSE and Back-Propagation (BP). This research on the topic of energy performance of buildings has been recently raising concerns about energy waste. The computation of the heating and cooling load to perform the efficient building design is required to determine the specifications of the heating and cooling equipment needed to maintain comfortable indoor air conditions $[17,18]$. The experiments are performed on the estimation of energy performance of 768 diverse residential buildings.

This paper is organized in the following fashion. In Section 2, the procedure steps of CFCM clustering methods are described. The entire design concept and process of IRBFN are proposed in Section 3. The experimental results are performed and discussed in Section 4. Concluding comments are covered in Section 5.

\section{Context-Based Fuzzy C-Means Clustering}

The CFCM clustering introduced by Pedrycz [8] estimates the cluster centers preserving homogeneity with the use of fuzzy granulation. First, the contexts are produced from the output variable used in the modeling problem. Next, the cluster centers are estimated by FCM clustering from input data points included in each context. By forming fuzzy clusters in input and output spaces, CFCM clustering converts numerical data into semantically meaningful information granules.

In what follows, we briefly describe the essence of CFCM clustering [8]. In a batch-mode operation, this clustering determines the cluster centers and the membership matrix by the following steps.

Step 1. Select the number of contexts $p$ and cluster center $c$ in each context, respectively.

Step 2. Produce the contexts in output space. These contexts were generated through a series of triangular membership functions equally spaced along the domain of an output variable. However, we may encounter a data scarcity problem due to small data included in some linguistic context. Thus, this problem brings about the difficulty to obtain clusters from the CFCM clustering. Therefore, we use probabilistic distribution of output variable to produce the flexible linguistic contexts [13].

Step 3. Once the contexts have been formed, the clustering is directed by the provided fuzzy set of each context.

Step 4. Initialize the membership matrix with random values between 0 and 1 .

Step 5. Compute $c$ fuzzy cluster centers using (1).

Here, fuzzification factor is generally used as fixed value $m=2$.

$$
\mathbf{v}_{i}=\frac{\sum_{k=1}^{N} u_{i k}^{m} \mathbf{x}_{k}}{\sum_{k=1}^{N} u_{i k}^{m}}
$$

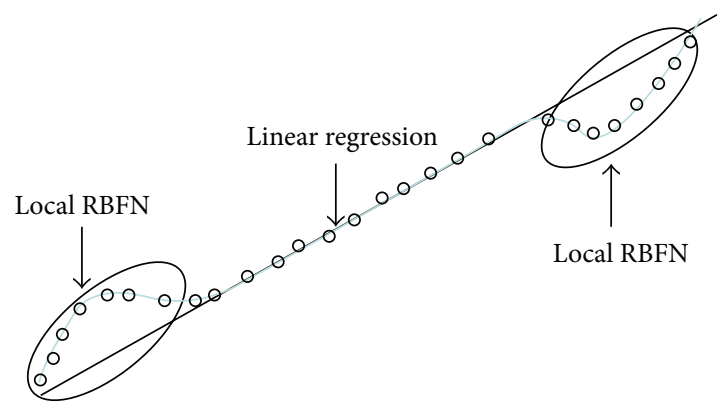

FIgURE 1: Combination of LR and local RBFN in the design of incremental RBFN (o: data points).

Step 6. Compute the objective function according to

$$
J=\sum_{i=1}^{c} \sum_{k=1}^{N} u_{i k}^{m} d_{i k}^{2}
$$

where $d_{i k}$ is the Euclidean distance between $i^{\prime}$ th cluster center and $k^{\prime}$ th data point. The minimization of objective function is obtained by iteratively updating the values of the membership matrix and cluster centers. Stop if it is below a certain tolerance value.

Step 7. Compute a new membership matrix using (3). Go to Step 5.

$$
u_{t i k}=\frac{w_{t k}}{\sum_{j=1}^{c}\left(\left\|\mathbf{x}_{k}-\mathbf{v}_{i}\right\| /\left\|\mathbf{x}_{k}-\mathbf{v}_{j}\right\|\right)^{2 /(m-1)}},
$$

where $u_{t i k}$ represents the element of the membership matrix induced by the $i$ th cluster and $k$ th data in the $t$ th context. $w_{t k}$ denotes a membership value of the $k$ th data point included by the $t$ th context.

\section{Incremental Radial Basis Function Networks (IRBFN)}

In this Section, we focus on two essential phases of the proposed IRBFN (Incremental RBFN) as underlying principle. First, we design a standard LR which could be treated as a preliminary construct capturing the linear part of the data. Next, the local RBFN is designed to eliminate errors produced by the regression part of the model. Figure 1 shows the example of nonlinear relationships and their modeling through a combination of LR model of a global character and a collection of local RBFN. As shown in Figure 1, the Linear Regression exhibits a good match except for two local areas. These remaining regions are predicted by local RBFN with the use of information granules through CFCM clustering algorithm. Figure 2 shows the architecture and overall flow of processing realized in the design of the proposed IRBFN. steps.

The principle of the IRBFN is explained in the following

Step 1. Design of Linear Regression (LR) model in the inputoutput space: $z=\mathrm{L}(\mathbf{x}, \mathbf{b})$ with $\mathbf{b}$ denoting a vector of the 


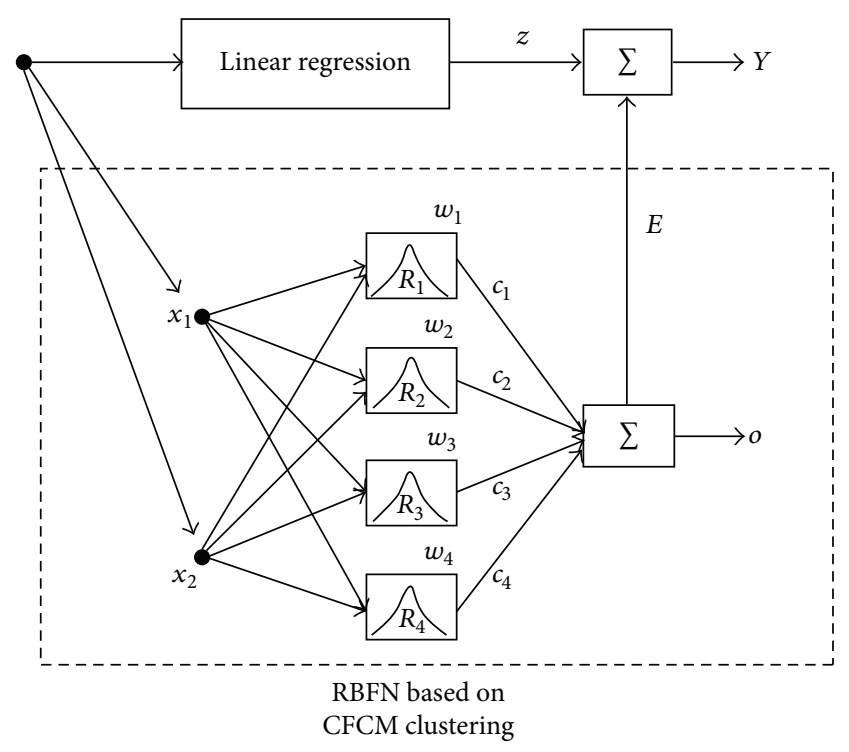

FIGURE 2: Main design process of incremental RBFN.

regression hyperplane of the linear model, $\mathbf{b}=\left[\begin{array}{ll}\mathbf{a} & a_{0}\end{array}\right]^{T}$; thus, we obtain the predicted output by using LR as a global model [6]; on the basis of the original data set, a collection of inputerror pairs is formed, $\left(\mathbf{x}_{k}, e_{k}\right)$.

Step 2. Construction of the collection of contexts in the error of the regression model $\left(E_{1}, E_{2}, \ldots, E_{p}\right)$ : here $p$ is the number of contexts; the distribution of these fuzzy sets is obtained through statistical method [7,8] mentioned in Section 2; the contexts are characterized by triangular membership functions with a 0.5 overlap between neighboring fuzzy sets.

Step 3. CFCM clustering completed in the input-output space from the contexts produced in the error space: the obtained cluster centers are used as the centers of receptive fields in the design of local RBFN as shown in Figure 1; for $p$ contexts and $c$ clusters for each context, the number of nodes in hidden layer is $c \times p$.

Step 4. Calculation of output in the design of local RBFN: the final output of RBFN is the weighted sum of the output value associated with each receptive field as follows:

$$
o=\sum_{i=1}^{c \times p} w_{i} c_{i}
$$

The receptive field functions are fixed, and then the weights of the output layer are directly estimated by LSE (Least Square Estimate) and BP (Back-Propagation). These methods are known as the most representative techniques frequently used in conjunction with RBFN [2]. In order to learn and adapt the architecture of RBFN to cope with changing environments, we need BP learning, if we use the steepest descent method to tune the centers of radial basis function and the output weights in the design of RBFN. Otherwise, we can directly obtain the output weights as one-pass estimation using LSE.
TABLE 1: Optimal values of the fuzzification coefficient for selected number of contexts $(p)$ and clusters $(c)$.

\begin{tabular}{ccccc}
\hline$p$ & & $c$ & & \\
& 3 & 4 & 5 & 6 \\
\hline 3 & 2.6 & 2.1 & 2.2 & 2.3 \\
4 & 2.2 & 2.1 & 2.2 & 2 \\
5 & 1.9 & 1.9 & 1.9 & 2 \\
6 & 1.7 & 1.8 & 1.8 & 2 \\
\hline
\end{tabular}

Step 5. Calculation of final output of the proposed IRBFN: the granular result of the IRBFN is combined with the output of the linear part:

$$
Y=z+E
$$

In order to evaluate the overall performance, we use standard root mean square error (RMSE) defined as follows:

$$
\mathrm{RMSE}=\sqrt{\frac{1}{N} \sum_{k=1}^{N}\left(Y_{k}-y_{k}\right)^{2}}
$$

\section{Experimental Results}

In the experiments, we report on the design and performance of the proposed models to assess the heating load and cooling load requirements of building as a function of building parameters. All experiments were completed in the 10 -fold cross-validation mode with a typical $60 \%-40 \%$ split between the training and testing data subsets. We perform energy analysis using 12 different building shapes simulated in Ecotect $[17,18]$.

These 12 building forms were generated by taking the elementary cube $(3.5 \times 3.5 \times 3.5)$ where each building form is composed of 18 elements. The materials used for each elementary cube are the same for all building forms. The selection was made by the newest and most common materials in the building construction industry and by the lowest $U$-value [17]. The buildings differ with respect to the glazing area, the glazing area distribution, orientation, overall height, roof area, wall area, surface area, and relative compactness. The data set comprises 768 samples and 8 features. The attributes to be predicted in terms of the preceding 8 input attributes are two real valued responses (heating load and cooling load).

We obtained the experimental results with the two essential parameters $(p, c)$ controlling the granularity of the construct in the input and output space. The numerical range of the fuzzification factor $(m)$ used in the experiments is between 1.5 and 3.0 with the incremental step of 0.1 . Table 1 listed the optimal values of the fuzzification factor by the increase of the number of contexts and clusters. Figure 3 shows the variation of the RMSE caused by the fuzzification factor in the case of $p=c=6$ for heating load prediction. Here the optimal values of the parameters are such that the testing error becomes minimal.

In the conventional method [14], the contexts were produced through triangular membership functions, equally 


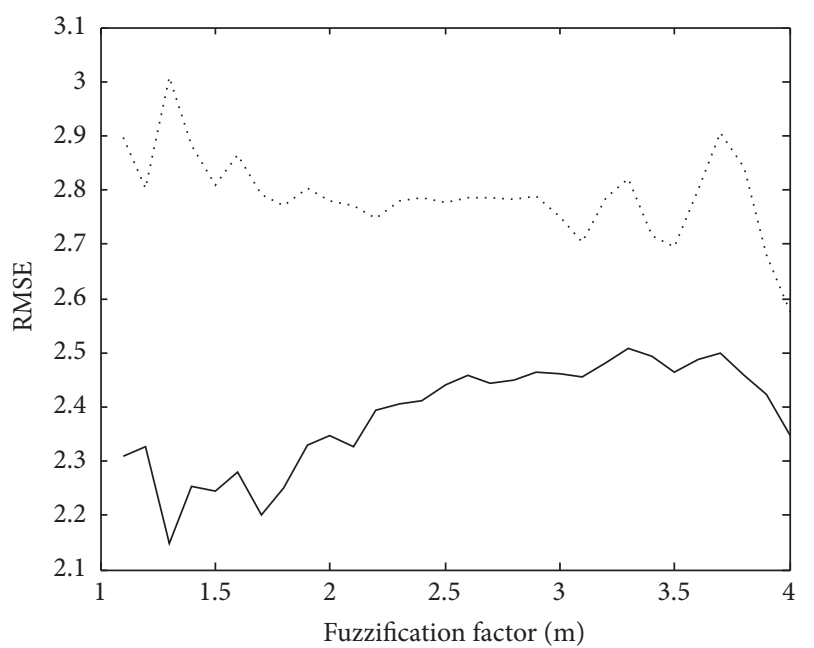

MSE (training)

RMSE (testing)

FIGURE 3: RMSE variation by the values of the fuzzification coefficient $(p=c=6)$.

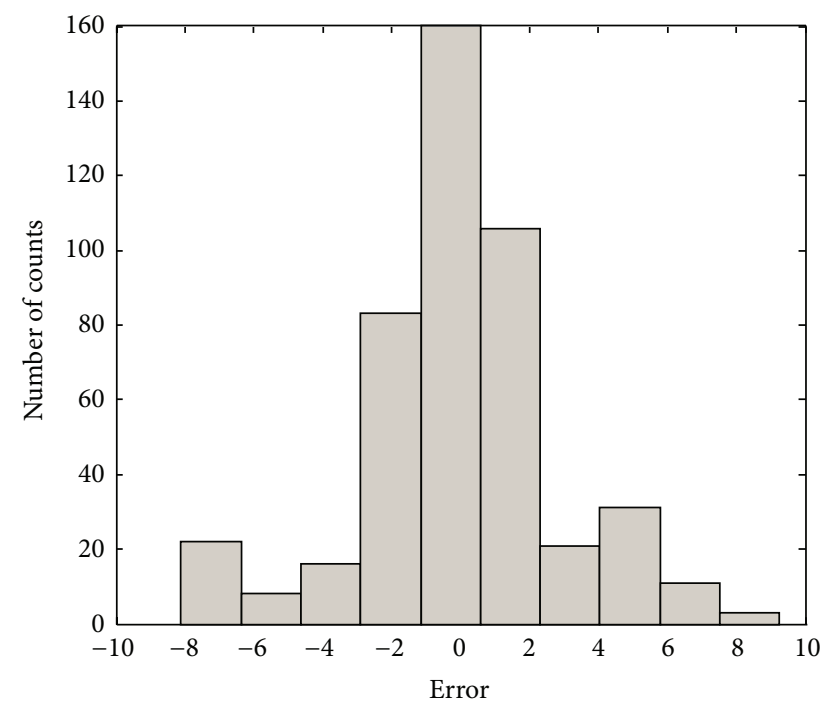

FIgURE 4: Histogram in the error space.

spaced along the domain of an output variable. However, we may encounter a data scarcity problem due to small amounts of data included in some context. Thus, we use a probabilistic distribution of the output variable to obtain flexible contexts. For this, the contexts in the error space are produced based on a histogram shown in Figure 4, Probability Density Function (PDF), and Conditional Density Function (CDF) [13]. Figure 5 shows the contexts $(p=6)$ generated in the error space of LR model as one example among 10fold cross-validation mode. Figure 6 shows the prediction performance based on local RBFN for the error of LR. As shown in Figure 6, the result clearly shows that the local RBFN with the use of information granulation has good prediction capability. Figures 7 and 8 show the performance

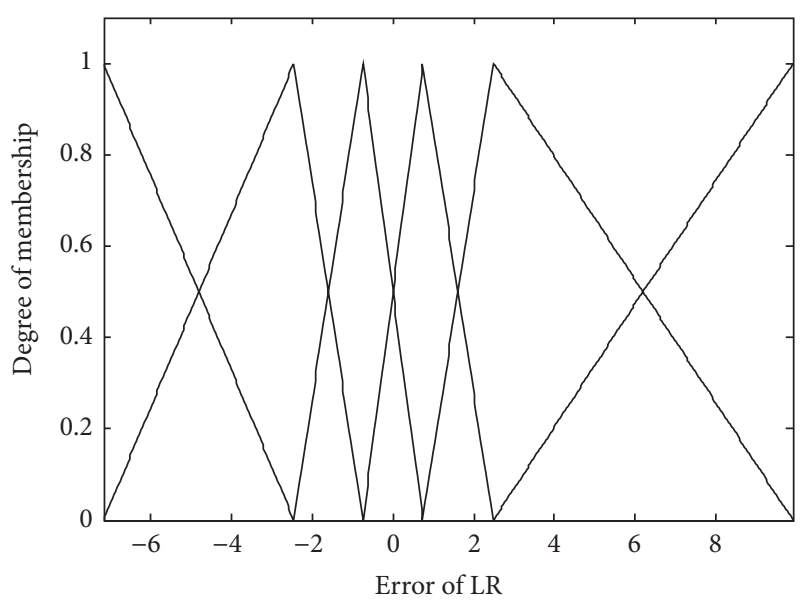

FIgURE 5: Contexts generated in the error space.

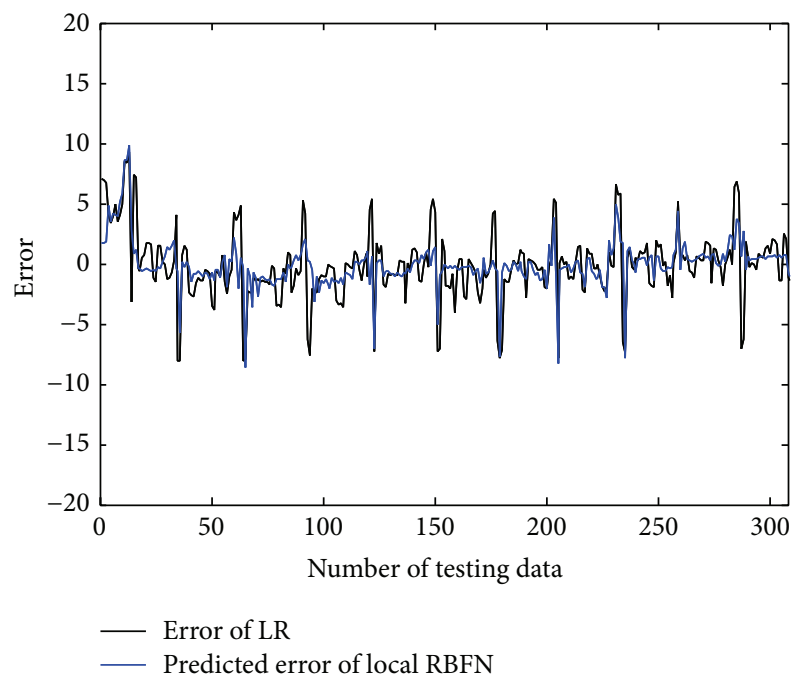

FIgURE 6: Prediction by local RBFN for the error of LR.

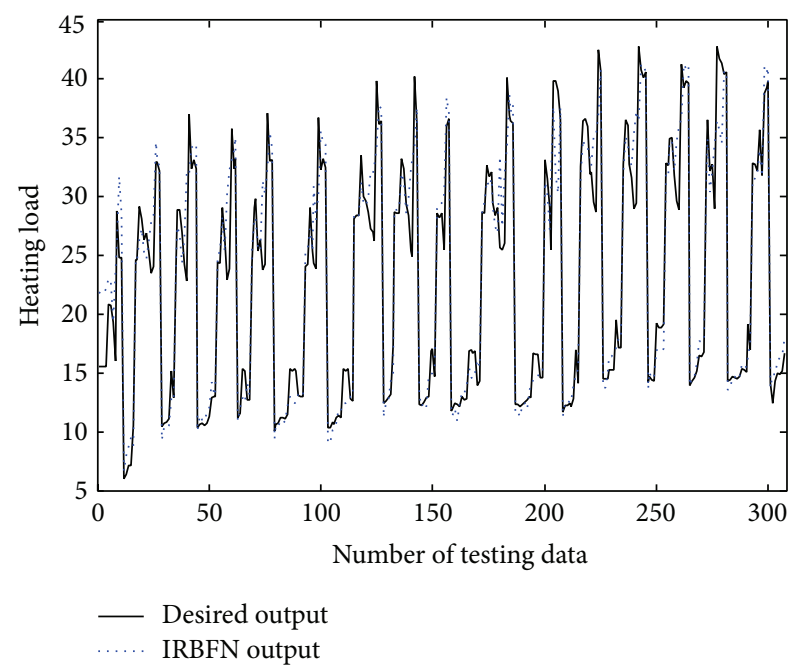

FIgURE 7: Prediction performance for heating load. 


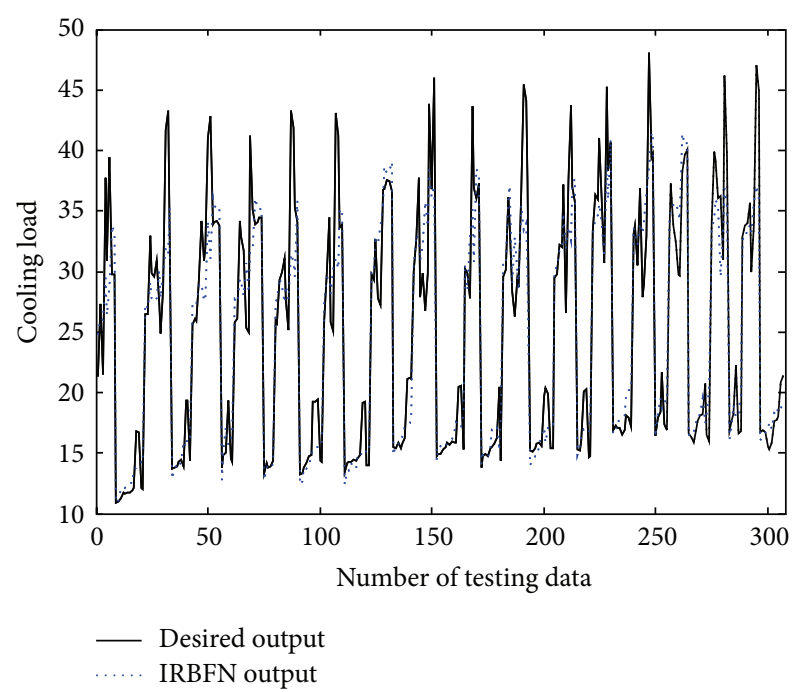

Figure 8: Prediction performance for cooling load.

TABLE 2: Comparison results of RMSE for the prediction of heating load (mean value of 10 -fold cross-validation) ( $*$ : number of rules).

\begin{tabular}{lccc}
\hline Methods & $\begin{array}{c}\text { Number of } \\
\text { nodes }\end{array}$ & $\begin{array}{c}\text { RMSE } \\
\text { (training) }\end{array}$ & $\begin{array}{c}\text { RMSE } \\
\text { (testing) }\end{array}$ \\
\hline LR & - & 2.936 & 2.911 \\
MLP & 36 & 2.883 & 2.890 \\
RBFN & 36 & 3.707 & 5.199 \\
RBFN (CFCM) [14] & 36 & 2.767 & 3.106 \\
LM & $36^{*}$ & 4.084 & 4.388 \\
Proposed incremental & & & \\
method & & & \\
$\quad$ IRBFN_LSE & 36 & 2.284 & 2.826 \\
$\quad$ IRBFN_BP & 36 & 2.353 & 2.730 \\
\hline
\end{tabular}

TABLE 3: Comparison results of RMSE for the prediction of cooling load (mean value of 10 -fold cross-validation) ( $*$ : number of rules).

\begin{tabular}{lccc}
\hline Methods & $\begin{array}{c}\text { Number of } \\
\text { nodes }\end{array}$ & $\begin{array}{c}\text { RMSE } \\
\text { (training) }\end{array}$ & $\begin{array}{c}\text { RMSE } \\
\text { (testing) }\end{array}$ \\
\hline LR & - & 3.180 & 3.208 \\
MLP & 36 & 3.176 & 3.226 \\
RBFN & 36 & 3.601 & 4.812 \\
RBFN (CFCM) [14] & 36 & 2.866 & 3.388 \\
LM & $36^{*}$ & 3.866 & 4.296 \\
Proposed incremental & & & \\
method & & & \\
$\quad$ IRBFN_LSE & 36 & 2.462 & 3.102 \\
$\quad$ IRBFN_BP & 36 & 2.555 & 3.089 \\
\hline
\end{tabular}

of IRBFN based on LSE for the prediction of heating load and cooling load, respectively. Here the number of epochs is 1000 and learning rate is 0.01 , respectively. As shown in these figures, the proposed IRBFN showed good generalization capability for testing data set, respectively. Tables 2 and 3 listed the comparison results of RMSE for the prediction of heating and cooling load, respectively. As listed in these tables, the experimental results revealed that the proposed IRBFN showed good performance in comparison to LR, MLP (Multilayer Perceptron), the conventional RBFN, RBFN with CFCM clustering, and LM (Linguistic Model).

\section{Conclusions}

We developed the incremental RBFN by combining LR and local RBFN for the prediction of heating load and cooling load of residential buildings. It was found from the result that the proposed IRBFN has good approximation and generalization capabilities with the aid of information granulation. These results lead us to the conclusion that the proposed IRBFN combined by LR and local RBFN showed a good performance in comparison to the previous works. For further research, we shall design this model to optimize the number of contexts and clusters per context based on evolutionary algorithm.

\section{Competing Interests}

The authors declare that they have no competing interests.

\section{Acknowledgments}

This work was supported by research funds from Chosun University, 2012.

\section{References}

[1] D. Simon, Evolutionary Optimization Algorithms, John Wiley \& Sons, 2013.

[2] J. S. R. Jang, C. T. Sun, and E. Mizutani, Neuro-Fuzzy and Soft Computing: A Computational Approach to Learning and Machine Intelligence, Prentice Hall, New York, NY, USA, 1997.

[3] S. Sumathi and S. Panneerselvam, Computational Intelligence Paradigms: Theory and Applications Using MATLAB, CRC Press, New York, NY, USA, 2010.

[4] T. P. Trappenberg, Fundamentals of Computational Neuroscience, Oxford University Press, Oxford, UK, 2nd edition, 2010.

[5] G. A. F. Seber, Linear Regression Analysis, Wiley Series in Probability and Mathematical Statistics, John Wiley \& Sons, New York, NY, USA, 1977.

[6] W. Pedrycz and K.-C. Kwak, "The development of incremental models," IEEE Transactions on Fuzzy Systems, vol. 15, no. 3, pp. 507-518, 2007.

[7] W. Pedrycz and F. Gomide, Fuzzy Systems Engineering Toward Human-Centric Computing, Wiley-Interscience, 2007.

[8] W. Pedrycz, "Conditional fuzzy C-means," Pattern Recognition Letters, vol. 17, no. 6, pp. 625-631, 1996.

[9] L. Hu and K. C. Chan, "Fuzzy clustering in a complex network based on content relevance and link structures," IEEE Transactions on Fuzzy Systems, vol. 24, no. 2, pp. 456-470, 2016.

[10] P. Fazendeiro and J. V. De Oliveira, "Observer-biased fuzzy clustering," IEEE Transactions on Fuzzy Systems, vol. 23, no. 1, pp. 85-97, 2015.

[11] T. C. Glenn, A. Zare, and P. D. Gader, "Bayesian fuzzy clustering," IEEE Transactions on Fuzzy Systems, vol. 23, no. 5, pp. 1545-1561, 2015. 
[12] A. Proietti, L. Liparulo, and M. Panella, " 2 D hierarchical fuzzy clustering using kernel-based membership functions," Electronics Letters, vol. 52, no. 3, pp. 193-195, 2016.

[13] S.-S. Kim and K.-C. Kwak, "Development of quantum-based adaptive neuro-fuzzy networks," IEEE Transactions on Systems, Man, and Cybernetics, Part B: Cybernetics, vol. 40, no. 1, pp. 91100, 2010.

[14] W. Pedrycz and A. V. Vasilakos, "Linguistic models and linguistic modeling," IEEE Transactions on Systems, Man, and Cybernetics Part B: Cybernetics, vol. 29, no. 6, pp. 745-757, 1999.

[15] K.-C. Kwak, "A design of genetically optimized linguistic models," IEICE Transactions on Information and Systems, vol. E95-D, no. 12, pp. 3117-3120, 2012.

[16] W. Pedrycz, "Conditional fuzzy clustering in the design of radial basis function neural networks," IEEE Transactions on Neural Networks, vol. 9, no. 4, pp. 601-612, 1998.

[17] L. Pérez-Lombard, J. Ortiz, and C. Pout, "A review on buildings energy consumption information," Energy and Buildings, vol. 40, no. 3, pp. 394-398, 2008.

[18] A. Tsanas and A. Xifara, "Accurate quantitative estimation of energy performance of residential buildings using statistical machine learning tools," Energy and Buildings, vol. 49, pp. 560$567,2012$. 

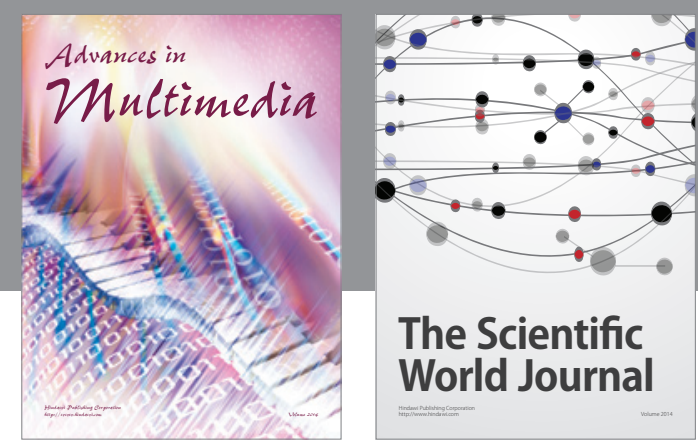

The Scientific World Journal
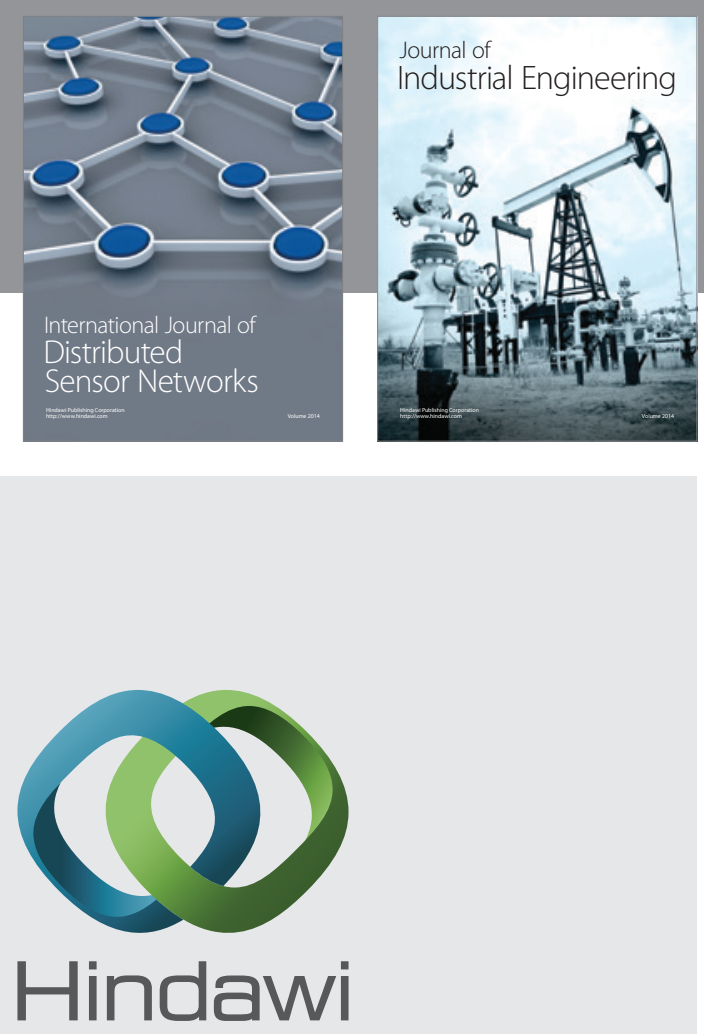

Submit your manuscripts at

http://www.hindawi.com

\section{Computer Networks} and Communications
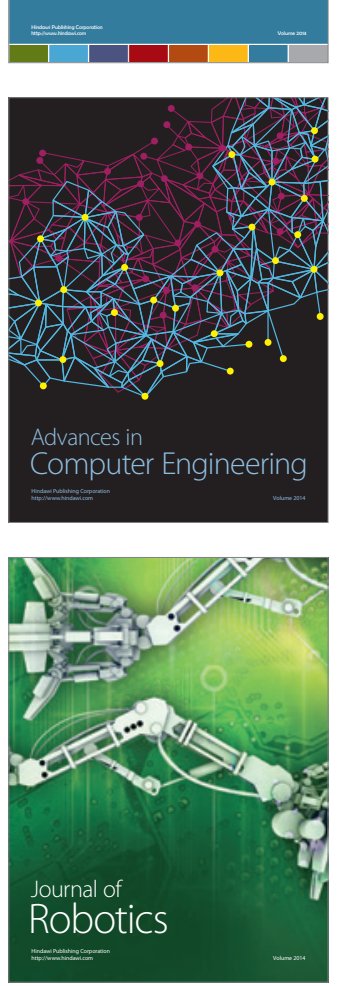
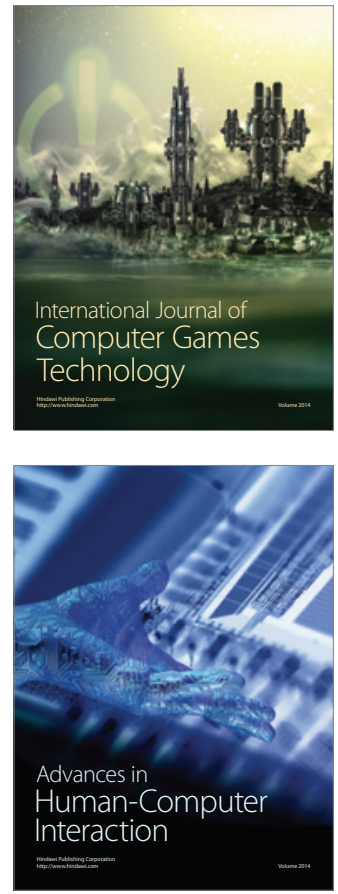
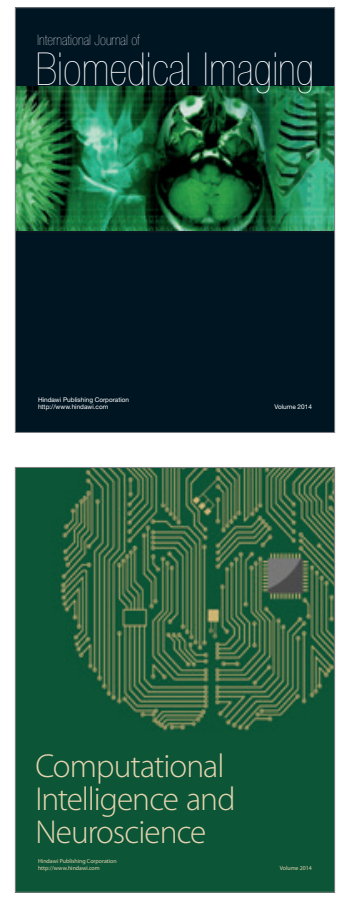
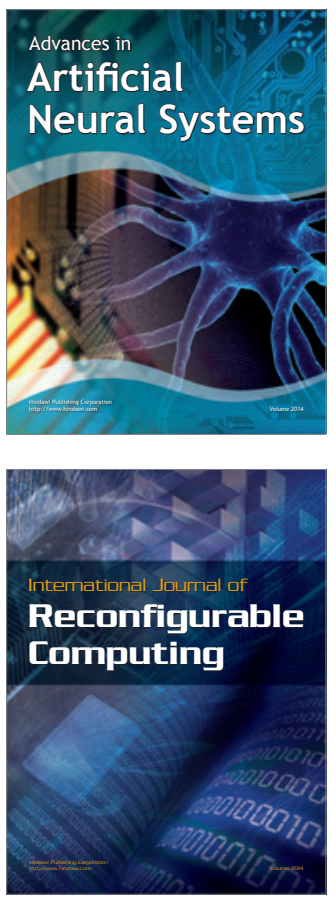
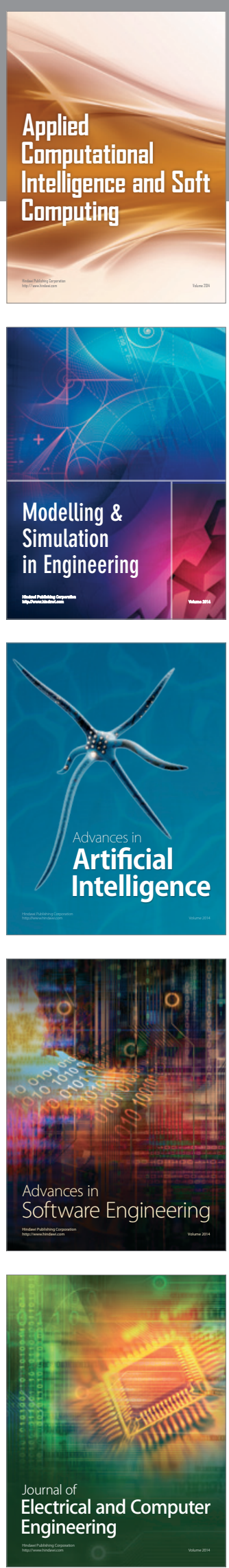